a role in using these clusters in technological devices. To address this issue, researchers from the Chinese Academy of Sciences, the National Research Council of Canada, Tohoku University, and the National Renewable Energy Laboratory have devised a way to create ordered, two-dimensional arrays of $\mathrm{Al}_{6}$ clusters on $\mathrm{Si}(111)$ surfaces.

They report in the April 29 issue of Applied Physics Letters that they formed nanocluster arrays by evaporating $\mathrm{Al}$ on a $\operatorname{Si}(111) 7 \times 7$ surface. At substrate temperatures above $200^{\circ} \mathrm{C}$, an ordered array of uniform Al clusters is formed. The arrays were studied with scanning tunneling microscopy, which showed that the clusters contain six $\mathrm{Al}$ atoms, occupying both halves of the $\operatorname{Si}(111) 7 \times 7$ unit cell, giving the nanocluster array the same geometry as the underlying $7 \times 7$ substrate. First-principles total-energy calculations find an optimized structure for the $\mathrm{Al}_{6}$ clusters, which is in agreement with the experimental images.

"The formation of uniform $\mathrm{Al}$ and other metal cluster arrays provides new practical approaches for doping Si with ultrahigh uniformity and atomic precision," said Qi Kun Xue, the lead researcher on the project. "Precision doping also opens the door for fabricating $p-n$ junctions across the two halves of each of the $\mathrm{Si}(111) 7 \times 7$ unit cells for diode applications. This would probably be among the highest density of integrated solidstate devices that may, one day, reach the commercial market. We are now working in collaboration with the Rowland Institute at Harvard University on proving this very exciting idea," Xue said.

The Al clusters are the most stable metal clusters that the research team has grown. The fact that the Al cluster arrays can sustain temperatures of up to $500^{\circ} \mathrm{C}$ is an important factor for practical applications, they said. The researchers also believe the $\mathrm{Al}_{6}$-decorated $\mathrm{Si}(111)$ surface could serve as a template for the growth of other types of nanocluster arrays.

CHRISTOPHER MATRANGA

\section{High-Efficiency Solar-Blind GaN Photocathodes Produced for Low-Level UV Detection}

Researchers Bruce Wessels, Melville Ulmer, and colleagues at Northwestern University have fabricated a solar-blind UV detector based on a Cs-treated $p$-GaN photocathode. The device achieved a relatively high quantum efficiency (QE) of 30\% for detecting radiation in the deep UV $(\lambda=$ $200 \mathrm{~nm}$ ), and a UV-visible light-rejection ratio of about 4 orders of magnitude.

Solar blindness is a necessity in several
UV detector applications, for example, in UV astronomy, where objects studied are usually 4-8 orders of magnitude brighter in the visible than in the UV range. One of the approaches to making such UV-sensitive photodetectors is by using III-nitride materials as photocathodes by taking advantage of their negative electron affinity (NEA). NEA is obtained when the minimum of the semiconductor conduction band is above the vacuum level. In that case, thermalized electrons can escape the surface of the bulk material. To attain the NEA, the surface of the semiconductor must be treated with impurities.

As reported in the April issue of the IEEE Journal of Quantum Electronics, the researchers grew GaN layers $1 \mu \mathrm{m}$ thick on sapphire substrates by metalorganic chemical vapor deposition. Magnesium was used as the $p$-type dopant, and films were treated with cesium to achieve NEA. In the as-grown materials, carrier concentrations were in the range of $5 \times$ $10^{16} \mathrm{~cm}^{-3}$ to $1 \times 10^{17} \mathrm{~cm}^{-3}$ and mobilities were in the range of $3-11.5 \mathrm{~cm}^{2} / \mathrm{V} \mathrm{s}$. Subsequently, the films were processed into photocathode tubes.

In the sample with the best material quality, the researchers obtained a QE as high as $30 \%$ at $200 \mathrm{~nm}$ with a rejection ratio of about 4 orders of magnitude between the UV $(200 \mathrm{~nm})$ and visible $(500 \mathrm{~nm})$ ranges. They also found that the $\mathrm{QE}$
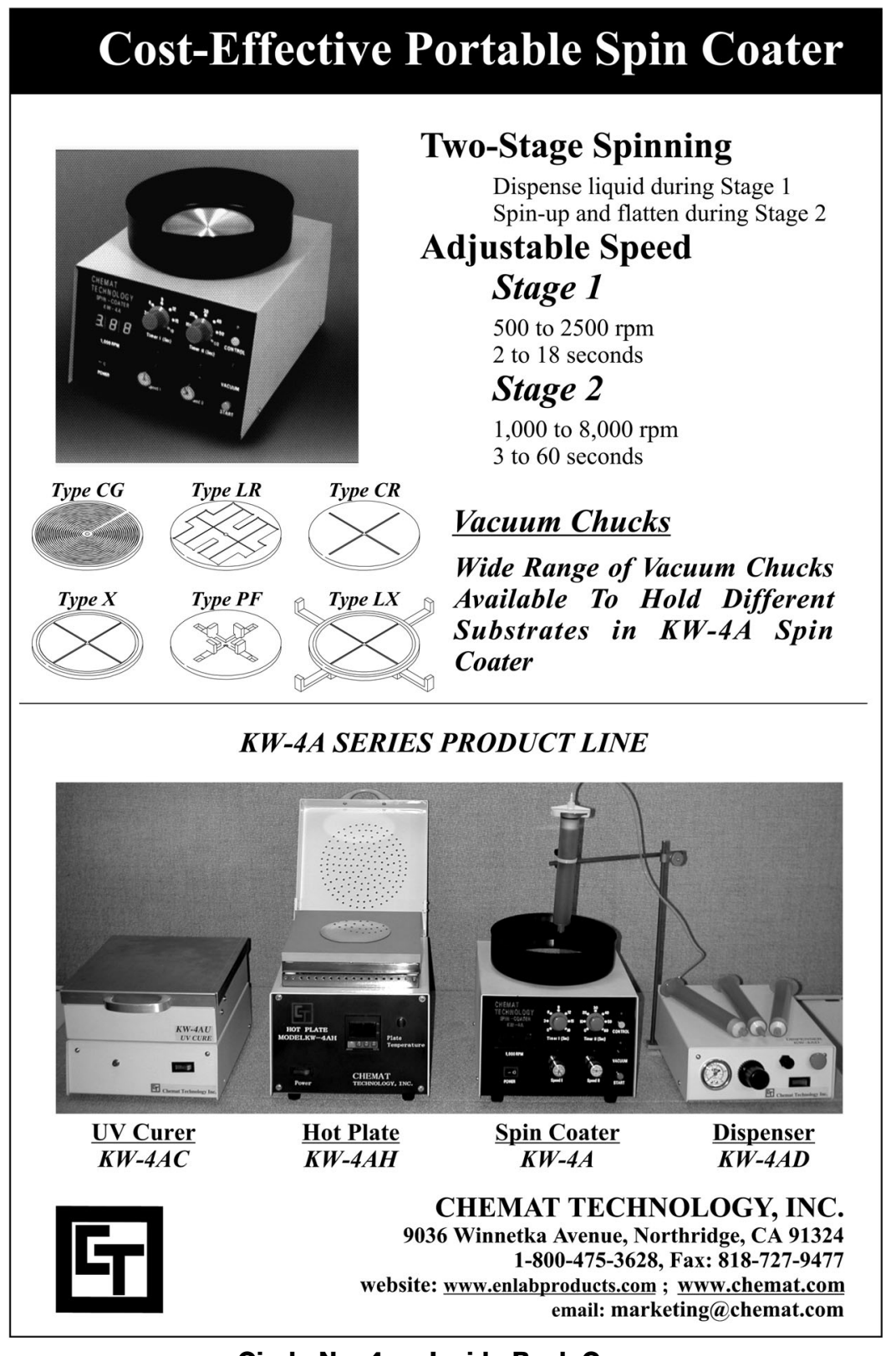

Circle No. 4 on Inside Back Cover 
increases with an increase in conductivity. Based on the findings, the researchers anticipate that a further improvement in the photocathode quantum efficiency can be achieved with higher conductivity of the material. Although a $30 \% \mathrm{QE}$ is still lower than QEs reported in the literature for the deep-UV (near $280 \mathrm{~nm}$ and below), the promise of achieving higher responsivity in a photocathode-type detector exists. Also, the photocathode tubes showed high stability over time.

The researchers are now working to improve the conductivity of this material to increase its efficiency as well as to improve the solar blindness of the resulting detectors.

"By adding electrically active impurities to gallium nitride, we have improved the optical properties of the material, making it very sensitive to ultraviolet light, but we know we can do even better," said Wessels. "Our goal is to make the device with greater than $50 \%$ quantum efficiency, which means it will be 10 times better than the detectors used in the Hubble Space Telescope." Quantum efficiencies as high as $90 \%$ are theoretically possible, according to the research team.

SHIMING WU

\section{Polymeric Material Self-Repairs Without Addition of Chemicals}

While many plastic materials are strong and resistant to breaking, once fractured, the broken pieces are cracked irreversibly. Although progress has been made in the development of self-healing polymers with the use of polymer catalysts or glues, further questions still remain concerning the long-term stability of the catalyst and the ability of the material to self-heal multiple times. Fred Wudl, professor of organic chemistry at the University of California-Los Angeles and director of UCLA's Exotic Materials Institute, led a research group of chemists and engineers who developed a transparent plastic that, when fractured, mends itself with heating.

Unlike other self-healing plastics developed previously, this material, called automend, repairs itself without the addition of a catalyst or other chemicals. As reported in the March 1 issue of Science, automend is an extremely hard and fully transparent polymer material prepared by the Diels-Alder cycloaddition of a multi-diene and a multi-dienophile. Its density is $1.37 \mathrm{~g} / \mathrm{cm}^{3}$ with an index of refraction of 1.56 at $24^{\circ} \mathrm{C}$, its Young's modulus is $4.72 \mathrm{GPa}$, and its Poisson ratio is 0.349 . Its mechanical properties are similar to commercial epoxy resins.

Automend can be fractured and healed multiple times through heating at $120^{\circ} \mathrm{C}$, due to reversible cross-linking covalent bonds formed between the polymer chains and branches. While being heated, these bonds between the polymer chains are broken; but during cooling, the polymer will be remanufactured by reforming those broken bonds. Solid-state nuclear magnetic resonance spectroscopy showed that when the temperature is above $120^{\circ} \mathrm{C}, \sim 30 \%$ of intermonomer linkages disconnect and then reconnect upon cooling. After healing, the original fracture is invisible, according to scanning electron microscope images, and the polymer can retain $\sim 60 \%$ of its original fracture strength from the fracture tests.

Although this new polymeric structuralengineering material has potential use in large lenses and housings for radar or communications equipment, the researchers addressed some future studies for improvement such as the maleimide monomer's high melting temperature, curing time of the material, and service temperature range.

YuE Hu

\section{Transport Properties Measured by Hybrid Molecular-Assembly Approach}

Progress in the development of nanoscale molecular electronics requires the rapid screening of molecular systems for their electronic-transport properties. An essential element of this process involves the connection of electrodes to the molecules of interest. A team of researchers led by Raymond K. Tsui in the Physical Sciences Research Laboratories at Motorola Laboratories in Tempe, Ariz., has devised a hybrid molecular-assembly technique to facilitate the rapid screening of candidate molecules: first, forming a self-assembled monolayer (SAM) on a pair of prepatterned electrodes, then using metallic nanoparticles for gap-bridging under an ac field as an alternative fabrication approach to provide a solution for transport measurements of molecules.

As reported in the April 15 issue of Applied Physics Letters, the researchers fabricated gold nanoparticle electrodes using a combination of photolithography and electron-beam lithography on an oxidized Si substrate. With the lift-off technique, fine features of the electrodes were patterned by a poly(methyl methyacrylate) (PMMA) resist process. After the substrate was cleaned to remove oxidized $\mathrm{Au}$, the researchers assembled nanoparticles by applying an ac bias (0.5-2.5 V, peak-to-peak) ranging from $1 \mathrm{MHz}$ to $10 \mathrm{MHz}$ for 5-20 s. By applying an ac field, a dielectrophoretic force was creat- ed that pulled the nanoparticles in the direction of maximum field strength between the gap. The researchers measured various sizes of $\mathrm{Au}$ nanoparticles, ranging from $40 \mathrm{~nm}$ to $100 \mathrm{~nm}$, trapped between the gap with a yield of almost $100 \%$. They also discovered that as soon as the gold particle bridged the gap, the electric field in the gap was reduced, which tended to prevent additional accumulation. Additionally, since the resistance of the $\mathrm{Au}$ electrode-particle assembly varied from $100 \mathrm{k} \Omega$ to a few tens of megaohms, a larger number of nanoparticles could be clustered in the gap region if the nanoparticle size was smaller than the gap.

The researchers selected 2,5-diphenylethynyl-4',4"-dithioacetyl-nitrobenzene $\left(1^{\prime}\right)$ as a test molecule. The SAM tested consisted of a Au-1'-Au-1"-Au structure and showed qualitative current-voltage $(I-V)$ measurements with two negative differential resistance peaks similar to those observed in semiconductor heterostructures.

"Our approach is relatively simple and... can potentially allow a quick and easy way to characterize a large number of electronic molecules," said Islamshah Amlani, a senior engineer at Motorola Labs.

KINSON C. KAM

\section{Field-Emission Current Induces High Stable Temperature in Multiwalled Carbon Nanotubes}

S.T. Purcell and colleagues at the Université Claude Bernard Lyon 1 have induced stable temperatures of up to $2000 \mathrm{~K}$ in multiwalled carbon nanotubes (MWNTs) by field-emission current. As reported in the March 11 issue of Physical Review Letters, the researchers determined the temperature and resistance of individual MWNTs with field-emission electron spectroscopy. Both the high temperature and the light emission seen during field emission come from Joule heating of the MWNTs. The researchers propose that field emission can be used to improve the structure by heat treating. They also determined that the resistance of the MWNTs decreases with temperature.

The MWNTs were grown by chemical vapor deposition onto Ni tips. Scanning electron microscopy showed that the MWNTs were straight, $\sim 30 \mathrm{~nm}$ in diameter, and $\sim 40 \mu \mathrm{m}$ long. Transmission electron microscopy confirmed that they were multiwalled.

Field-emission experiments were conducted in ultrahigh vacuum of $\sim 7 \times 10^{-11}$ Torr. The total-energy distributions (TEDs) of the emitted electrons were measured with a hemispherical electronenergy analyzer. Although many MWNTs 